\title{
CCL2 Serum Levels and Adiposity Are Associated with the Polymorphic Phenotypes -2518A on CCL2 and 64ILE on CCR2 in a Mexican Population with Insulin Resistance
}

\author{
Milton-Omar Guzmán-Ornelas, ${ }^{1,2}$ Marcelo Heron Petri, ${ }^{1,3}$ \\ Mónica Vázquez-Del Mercado, ${ }^{1,4,5}$ Efraín Chavarría-Ávila, ${ }^{1,2,6}$ \\ Fernanda-Isadora Corona-Meraz, ${ }^{1,2}$ Sandra-Luz Ruíz-Quezada, ${ }^{2,7}$ \\ Perla-Monserrat Madrigal-Ruíz, ${ }^{1,2,4}$ Jorge Castro-Albarrán, ${ }^{2}$ \\ Flavio Sandoval-García, ${ }^{1}$ and Rosa-Elena Navarro-Hernández ${ }^{1,2,4,7}$ \\ ${ }^{1}$ Instituto de Investigación en Reumatología y del Sistema Musculo Esquelético, Centro Universitario de Ciencias de la Salud, \\ Universidad de Guadalajara, Sierra Mojada No. 950, Colonia Independencia, 44340 Guadalajara, JAL, Mexico \\ ${ }^{2}$ UDG-CA-701, Grupo de Investigación Inmunometabolismo en Enfermedades Emergentes (GIIEE), \\ Centro Universitario de Ciencias de la Salud, Universidad de Guadalajara, Sierra Mojada No. 950, Colonia Independencia, \\ 44340 Guadalajara, JAL, Mexico \\ ${ }^{3}$ Translational Cardiology, Center for Molecular Medicine, Department of Medicine, Karolinska Institutet, L8:03, \\ 17176 Stockholm, Sweden \\ ${ }^{4}$ Departamento de Biología Molecular y Genómica, Centro Universitario de Ciencias de la Salud, Universidad de Guadalajara, \\ Sierra Mojada No. 950, Colonia Independencia, 44340 Guadalajara, JAL, Mexico \\ ${ }^{5}$ Servicio de Reumatología, División de Medicina Interna, Hospital Civil "Dr. Juan I. Menchaca”, Universidad de Guadalajara, \\ Salvador de Quevedo y Zubieta No. 750, 44340 Guadalajara, JAL, Mexico \\ ${ }^{6}$ Departamento de Disciplinas Filosófico, Metodológico e Instrumentales, Centro Universitario de Ciencias de la Salud, \\ Universidad de Guadalajara, Sierra Mojada No. 950, Colonia Independencia, 44340 Guadalajara, JAL, Mexico \\ ${ }^{7}$ Departamento de Farmacobiología, Centro Universitario de Ciencias Exactas e Ingenierías, Universidad de Guadalajara, \\ Boulevard Marcelino García Barragán No. 1421, 44430 Guadalajara, JAL, Mexico
}

Correspondence should be addressed to Rosa-Elena Navarro-Hernández; rosa_elena_n@hotmail.com

Received 17 July 2015; Revised 5 October 2015; Accepted 15 October 2015

Academic Editor: Michal Ciborowski

Copyright ( 2016 Milton-Omar Guzmán-Ornelas et al. This is an open access article distributed under the Creative Commons Attribution License, which permits unrestricted use, distribution, and reproduction in any medium, provided the original work is properly cited.

Genetic susceptibility has been described in insulin resistance (IR). Chemokine (C-C motif) ligand-2 (CCL2) is overexpressed in white adipose tissue and is the ligand of C-C motif receptor-2 (CCR2). The CCL2 G-2518A polymorphism is known to regulate gene expression, whereas the physiological effects of the CCR2Val64Ile polymorphism are unknown. The aim of the study is to investigate the relationship between these polymorphisms with soluble CCL2 levels (sCCL2), metabolic markers, and adiposity. In a cross-sectional study we included 380 Mexican-Mestizo individuals, classified with IR according to Stern criteria. Polymorphism was identified using PCR-RFLP/sequence-specific primers. Anthropometrics and metabolic markers were measured by routine methods and adipokines and sCCL2 by ELISA. The CCL2 polymorphism was associated with IR (polymorphic $A+$ phenotype frequencies were $70.9 \%, 82.6 \%$, in individuals with and without IR, resp.). Phenotype carriers CCL2 $(A+)$ displayed lower body mass and fat indexes, insulin and HOMA-IR, and higher adiponectin levels. Individuals with IR presented higher sCCL2 compared to individuals without IR and was associated with CCR2 (Ile+) phenotype. The double-polymorphic phenotype carriers $(A+/$ Ile +$)$ exhibited higher sCCL2 than double-wild-type phenotype carriers (A-/Ile-). The present findings suggest that sCCL2 production possibly will be associated with the adiposity and polymorphic phenotypes of CCL2 and CCR2, in Mexican-Mestizos with IR. 


\section{Introduction}

The insulin resistance (IR) presents many subclinical manifestations, characterized by alterations in lipids and carbohydrates metabolism at different levels. Most of these changes is due to a low-grade systemic chronic inflammation $[1,2]$. Adipose tissue is the primary anatomical site where the disease takes place. In early stage, this tissue became inflamed with the following pathological mechanism: first, monocytes migrate to adipose tissue, these cells express high levels of $\mathrm{C}$ $\mathrm{C}$ motif receptor 2 (CCR2) and release monocyte chemoattractant protein-1 (MCP-1), also known as chemokine (C$\mathrm{C}$ motif) ligand 2 (CCL2). This chemokine can promote further local inflammation and/or acts in paracrine way. The signaling through CCR2 may polarize the monocytes to M1 macrophages; these cells present a proinflammatory profile. Nevertheless, CCL2-CCR2 interaction is known to regulate continuous migration of monocytes to adipose tissue [3-5].

CCL2 is produced in soluble form by monocytes and macrophages and binds with high affinity to the CCR2 receptor. The later one is constitutively expressed in monocytes and its levels decrease as it differentiate into macrophages [4].

The human CCL2 gene is located on chromosome 17q11.2 $[6,7]$. It has two remote kappa $\mathrm{B}$ binding sites known as A1 $(-2640 /-2632)$ and A2 $(-2612 /-2603)$ that regulates the transcription of CCL2 gene. Whereas the CCR2 belongs to the family of seven transmembrane-spanning receptors that are coupled to heterotrimeric $\mathrm{G}$ proteins, the gene is located on the chromosome $3 \mathrm{p} 21$ within a cluster of chemokine receptor genes $[7,8]$.

The IR is considered a multifactor and polygenic disease; nevertheless, it has not determine the environmental and genetic factors contribution. In this context the study of candidate genes to make a contribution in clarifying this point is important. It has been reported that single nucleotide polymorphisms (SNP) in CCL2 and CCR2 are related with IR, G-2518A (rs 1024611), and Val64Ile (rs 17998649), respectively. The SNP of CCL2 is located at 85 base pairs (bp) of remote kappa $\mathrm{B}$ binding site $\mathrm{A} 2$, while in CCR2 gene, the SNP is conservative and the amino acid change (Val>Ile) takes place in the first transmembrane domain. This change decreases the affinity CCL2 binding, since the join of CCL2 with CCR2 receptor is through the second transmembrane domain [9-11].

Clinical phenotype has been described with these polymorphisms; type 2 diabetes mellitus (T2DM), cardiometabolic risk factors, obesity indexes, and insulin secretion association were found [12]. Interestingly, another study failed to show effect of this polymorphism in adipokines levels in patients with essential hypertension and T2DM [13]. Studies in animal models were performed to demonstrate the functional effect of these polymorphisms, but the results were not conclusive [14].

With this in mind, the aim of this study is to describe the presence of polymorphism of CCL 2 and CCR 2 in a population with IR, compared to healthy subjects, and to elucidate the clinical/metabolic features that these polymorphisms may present.

\section{Material and Methods}

2.1. Subjects' Assessment. In this cross-sectional study, a total of 380 nonrelated Mexican-Mestizos (i.e., an individual that were born in Mexico, with a Spanish last name and a family history of Mexican ancestors for at least three generations), and aged 20-69 years, were recruited from population of Western Mexico and classified according to Stern criteria in two groups: group 1 individuals with IR, if any of the following conditions were met: HOMA-IR $>4.65$ or BMI $>27.5 \mathrm{~kg} / \mathrm{m}^{2}$ and HOMA-IR > 3.6, and group 2 individuals without IR, therefore negative for those who did not meet the above conditions (i.e., HOMA-IR $\leq 4.65$ or BMI $\leq 27.5 \mathrm{~kg} / \mathrm{m}^{2}$ and HOMA-IR $\leq 3.60$ ) [15]. Inclusion criteria for the study were considered as follows: individuals who at the time of the study did not present glucose intolerance, infectious diseases, hypertension, history of cardiovascular disease, malignancy, and renal and metabolic diseases such as T2DM. The subjects were questioned and denied any medication or weight change at least 3 weeks.

2.2. Ethics Conduct. Before enrolment, participants were informed about the study and signed a consent form following the Helsinki declaration guidelines, and the institutional (Guadalajara University) review boards' committees ensured appropriate ethical and biosecurity conduct [16].

2.3. Medical History and Physical Examination. All individuals who fulfil inclusion criteria were clinically evaluated by a physician who performed a complete medical history and assessment of general health status and vital signs were included: blood pressure (executed 3 times with the subject in the sitting position and relaxing for 15 minutes before the measurement), heart and respiratory rate, and body temperature.

2.4. Body Fat Storage Measurements. We evaluated the following body measurements: height, measured to the nearest $1 \mathrm{~mm}$ by using a stadiometer (Seca GmbH \& Co. KG. Hamburg, Germany), weight, body mass index (BMI), and total body fat, determined by using bioelectrical impedance analysis (TANITA TBF304.Tokio, JPN) to the nearest $0.1 \mathrm{~kg}$. Waist and hip circumferences where measured to the nearest $0.1 \mathrm{~cm}$ by using an anthropometric fiberglass tape (GULICK length $0-180 \mathrm{~cm}$ precision $\pm 1 \mathrm{~mm}$; USA) following the procedures recommended by the anthropometric indicators measurement guide $[17,18]$. We calculated the waist-hip ratio [19] $[$ WHR $=$ waist $(\mathrm{cm}) /$ hip $(\mathrm{cm})]$, body fat ratio $[\mathrm{BFR}=$ body fat mass $(\mathrm{kg}) /$ height $\left.^{2}\left(\mathrm{~m}^{2}\right)\right]$, and waist to height ratio [WHtR = waist $(\mathrm{cm}) /$ height $(\mathrm{cm})$ ], as indicators of adiposity $[20,21]$. 
2.5. Laboratory Techniques and Procedures. Individuals included in the study were fasting 12 hours before the blood samples were taken, after allowing them to clot at room temperature; then the blood was centrifuged at 1509 RCF (Rotanta 460R, Andreas Hettich GmbH \& Co. KG) for 10 minutes at $20^{\circ} \mathrm{C}$. Serum was collected and stored at $-86^{\circ} \mathrm{C}$ until further analysis. We quantified the serum concentration of $\mathrm{C}$ reactive protein (CRP, with a limit of detection of $0.15 \mathrm{mg} / \mathrm{L}$ ), basal glucose, lipid profile that included triglycerides, total cholesterol, HDLc, LDLc, and VLDLc (high, low, and very low density lipoprotein cholesterol, resp.), and apolipoproteins A1 and B (ApoA1 and Apo-B, Randox Laboratories 55 Diamond Road, Crumlin Co. Antrim, Northern Ireland UK). By using commercial enzyme-linked immunoabsorbent assays (ELISA) were determined soluble levels of insulin (sensitivity of $0.399 \mu \mathrm{UI} / \mathrm{mL}$ ), sCCL2 (limit of detection of $2.3 \mathrm{pg} / \mathrm{mL}$ ), sAdiponectin (limit of detection $0.019 \mathrm{ng} / \mathrm{mL}$ ) (ALPCO Diagnostics 26-G Keewaydin Drive, Salem, NH), and sResistin (sensitivity $0.026 \mathrm{ng} / \mathrm{mL}$, R\&D Systems Inc., Minneapolis, MN, USA).

2.6. SNPs Analysis. Genomic DNA was obtained from total blood using a standard protocol for extraction with the modified Miller method as described previously [22] and was stored at $-20^{\circ} \mathrm{C}$ until being used for genotyping. For each gene studied, polymorphic regions were amplified by polymerase chain reaction (PCR) method as described previously $[23,24]$.

To analyze the CCR2 Val64Ile SNP was determined using sequence-specific primers (SSP): forward, Val 5' -TGGGCAACATGCTGGTCG-3', Ile $5^{\prime}$-TGGGCAACATGCTGGTCA-3', and reverse: $5^{\prime}$-TGGAAAATAAGGGCCACAGAC- $3^{\prime}$ annealing $62^{\circ} \mathrm{C}$, PCR product $413 \mathrm{bp}$ [11], and CCL2 G-2518A SNP, forward primer $5^{\prime}$-TCACGCCAGCACTGACCTCC- $3^{\prime}$, and reverse: $5^{\prime}$-ACTTCCCAGCTTTGCTGGCTGAG- $3^{\prime}$ with annealing temperature $56^{\circ} \mathrm{C}$, PCR product $250 \mathrm{bp}$.

The PCR were performed in a $25 \mu \mathrm{L}$ total volume (mixture with $100 \mathrm{ng}$ of DNA, $2 \mathrm{nM}$ of each primer, $0.20 \mathrm{mM}$ of each dNTP, $0.25 \mathrm{U}$ Taq polymerase, and 1x PCR buffer) and 1.5 or $3.0 \mathrm{mM}$ of $\mathrm{MgCl}_{2}$ for CCL2 or CCR2, respectively.

To determine CCL2 genotypes a PCR was performed and then a digestion of obtained products with $P V U$ II restriction enzyme. The lengths of fragments observed were as follows: 175 and 75 bp (allele A) and 250 bp (allele G). Electrophoresis was done at a constant voltage of $180 \mathrm{~V}$ on $6 \%$ polyacrylamide gels stained with silver nitrate. For quality control, a blank and samples previously confirmed as positive for each genotype were used as controls. To ensure the accuracy of genotype data, we used internal controls and repetitive experiments. In addition, both polymorphisms were identified in duplicate by two different analysts. The genotyping success rate was $100 \%$.

2.7. Statistical Analysis. Data were analyzed with the Statistics program SPSS v21 (IBM Inc., Chicago, IL, USA) and GraphPad Prism v6.01 (2014 Inc. 2236 Beach Avenue Jolla, CA 92037). Results are given as mean \pm SD or median with 25 ,
75 percentiles or percentages based on normal distribution. The data distribution of clinical and laboratory variables of the study group was evaluated with $Z$ Kolmogorov-Smirnov test, and we performed parametric and nonparametric test, as appropriate. The most important variables were adjusted by gender and age with an ANCOVA analysis. About these results we performed multifactorial analysis for the most important variables. The clinical and laboratory characteristics of the study group were performed with the unpaired Student's $t$-test or Mann-Whitney $U$ test, and to compare quantitative data in four groups, a one-way ANOVA and post hoc Tukey test were used.

Data from serum concentrations of adipokines, the laboratorial assessment, and disease variables were subjected to Pearson or Spearman correlation tests. The Hardy-Weinberg equilibrium text for individual loci was performed with http://ihg.gsf.de/cgi-bin/hw/hwal.pl. Contingency tables $(2 \times$ 2 and $2 \times 3$ ) with $\chi^{2}$ trend test or Fisher exact test, as appropriate, were used for testing the differences of genotype distribution and allele frequencies between study groups. Two genetic models were used for these analyses: (1) the dominant model where each SNP was modeled categorically and separated into three categories, one for each genotype, and (2) the phenotype model, where each SNP was modeled into two categories, with two genotypes combined into one category (polymorphic homozygotes plus heterozygotes), choosing one genotype (homozygotes wild type) as the reference group. A two-tailed $P$ value less than 0.05 was considered statistically significant.

\section{Results}

3.1. Adiposity Is Associated with Metabolic Markers. Anthropometrics characteristics and metabolic markers of the subjects included in this study split by IR Stern classification are shown in Table 1 . The study group included 380 MexicanMestizo individuals of which 237 (62\%) were women, they were classified without IR or with IR, ANCOVA was performed, adjusted for sex and age, and no differences were observed (data not showed). Twenty-one percent has been classified with obesity and 32\% with IR. Sixty-five percent of individuals, included in the study, were determined with excess body fat according to the Deurenberg criteria [21], and $31 \%$ had dyslipidemic profile (data no shown). A positive correlation of soluble levels of sCCL2, adipokines, metabolic markers, and lipid profile (except HDLc, LDLc, and Apo-Al) was observed along body fat storage (Table 2).

\subsection{Individuals with IR Presented Inflammatory State.} According to IR classification, individuals with IR displayed higher soluble levels of CCL2, resistin, and CRP and lower levels of adiponectin than individuals without IR (Figure 1). Soluble levels of CCL2, CRP, sResistin, and metabolic markers correlated positively with body adiposity, whereas levels of soluble adiponectin correlated negatively (Table 2 ).

3.3. CCL2 Polymorphism Is Associated with IR. All genotype frequencies were in Hardy-Weinberg equilibrium. 
TABLE 1: Anthropometric characteristics and metabolic markers in individuals included in the study.

\begin{tabular}{|c|c|c|c|}
\hline \multirow{2}{*}{ Measurement } & \multicolumn{2}{|c|}{ Study group } & \multirow{2}{*}{$P$} \\
\hline & Individuals without IR & Individuals with IR & \\
\hline$n(\%)$ & $270(68)$ & $110(32)$ & \\
\hline Age (years) & $35 \pm 14$ & $34 \pm 14$ & NS \\
\hline Height $(\mathrm{cm})$ & $163.8 \pm 5.7$ & $165.7 \pm 1.1$ & NS \\
\hline Weight (kg) & $68.7 \pm 13.2$ & $79.4 \pm 16.3$ & $<0.001^{*}$ \\
\hline BMI $\left(\mathrm{kg} / \mathrm{m}^{2}\right)$ & $24.9(22.6-28.9)$ & $28.6(24.4-31.7)$ & $<0.001^{+}$ \\
\hline Body fat mass (kg) & $20.1 \pm 9.2$ & $25.9 \pm 11.3$ & $<0.001^{*}$ \\
\hline Total body fat mass (\%) & $28.8 \pm 9.4$ & $32.80 \pm 9.2$ & $<0.001^{*}$ \\
\hline $\operatorname{BFR}\left(\mathrm{kg} / \mathrm{m}^{2}\right)$ & $7.03(5.24-9.40)$ & $9.40(6.37-12.20)$ & $0.001^{+}$ \\
\hline Waist circumference $(\mathrm{cm})$ & $86.5(77.1-93.1)$ & $95.0(83-103)$ & $<0.001^{+}$ \\
\hline Hip circumference $(\mathrm{cm})$ & $99.5(95-105.9)$ & $104(99-110)$ & $0.002^{+}$ \\
\hline WHR & $0.854(0.68-1.17)$ & $0.866(0.70-1.28)$ & $\mathrm{NS}^{+}$ \\
\hline WHtR & $0.533 \pm 0.076$ & $0.568 \pm 0.085$ & $<0.001^{*}$ \\
\hline Glucose (mg/dL) & $89 \pm 10$ & $98 \pm 18$ & $<0.001^{*}$ \\
\hline Insulin $(\mu \mathrm{UI} / \mathrm{mL})$ & $8.6 \pm 3.5$ & $35.2 \pm 32.5$ & $<0.001^{*}$ \\
\hline HOMA-IR & $1.86(1.34-2.58)$ & $6.05(4.40-9.03)$ & $<0.001^{+}$ \\
\hline Triglycerides (mg/dL) & $134 \pm 83$ & $162 \pm 95$ & $0.005^{*}$ \\
\hline Total cholesterol (mg/dL) & $184 \pm 40$ & $185 \pm 34$ & NS \\
\hline HDLc (mg/dL) & $39.5 \pm 15.1$ & $37.6 \pm 14.9$ & NS \\
\hline LDLc (mg/dL) & $111 \pm 36$ & $110 \pm 31$ & NS \\
\hline VLDLc (mg/dL) & $26 \pm 16$ & $32 \pm 19$ & $0.005^{*}$ \\
\hline Apo-A1 (mg/dL) & $114 \pm 25$ & $117 \pm 27$ & NS \\
\hline Apo-B (mg/dL) & $111 \pm 32$ & $117 \pm 32$ & NS \\
\hline
\end{tabular}

$n=380$. Data are presented as mean \pm standard deviation and median ( $25-75$ percentiles). ${ }^{*}$ Student's $t$-test and ${ }^{+}$Mann-Whitney $U$ test with $P$ significantly, comparing the groups: individuals with IR versus individuals without IR. IR: insulin resistance; BMI: body mass index; WHR: waist to hip ratio; WHtR: waist to height ratio; BFR: body fat ratio; HOMA-IR: homeostasis model assessment-insulin resistance; HDLc, LDLc, and VLDLc: high, low, and very low density lipoprotein cholesterol, respectively; Apo: apolipoprotein.

TABLE 2: Correlations soluble levels of adipokines and metabolic markers with body adiposity.

\begin{tabular}{|c|c|c|c|c|c|c|}
\hline \multirow{2}{*}{ Measurements } & ${ }^{*}$ Weight $(\mathrm{kg})$ & ${ }^{+} \mathrm{BMI}\left(\mathrm{kg} / \mathrm{m}^{2}\right)$ & ${ }^{+}$Body fat mass (\%) & ${ }^{+} \mathrm{BFR}\left(\mathrm{kg} / \mathrm{m}^{2}\right)$ & ${ }^{+} \mathrm{WC}(\mathrm{cm})$ & ${ }^{+} \mathrm{WHtR}$ \\
\hline & \multicolumn{6}{|c|}{$\%$ Correlation } \\
\hline $\mathrm{sCCL} 2(\mathrm{pg} / \mathrm{mL})$ & 20.1 & 29.4 & 21.8 & 29.3 & 25.0 & 24.8 \\
\hline CRP (mg/L) & 30.6 & 52.7 & 52.8 & 53.0 & 44.8 & 52.8 \\
\hline sAdiponectin (ng/mL) & -34.7 & -25.0 & -17.5 & -25.1 & -23.7 & -17.2 \\
\hline sResistin (ng/mL) & 43.3 & 27.1 & 30.1 & 27.3 & 32.5 & 24.9 \\
\hline Glucose (mg/dL) & 22.7 & 38.1 & 24.0 & 35.4 & 32.5 & 35.6 \\
\hline Insulin $(\mu \mathrm{UI} / \mathrm{mL})$ & 23.1 & 39.8 & 29.9 & 40.1 & 27.1 & 26.4 \\
\hline HOMA-IR & 25.0 & 43.6 & 32.4 & 43.5 & 30.5 & 30.6 \\
\hline Triglycerides (mg/dL) & 22.5 & 34.9 & 16.9 & 33.7 & 40.1 & 36.0 \\
\hline Total cholesterol (mg/dL) & 19.5 & 32.2 & 27.3 & 30.8 & 37.5 & 39.6 \\
\hline VLDLc (mg/dL) & 22.4 & 34.2 & 16.4 & 33.0 & 39.6 & 35.4 \\
\hline Apo-B (mg/dL) & 21.0 & 34.5 & 17.5 & 34.3 & 46.7 & 44.3 \\
\hline
\end{tabular}

$n=380$. BMI: body mass index; BFR: body fat ratio; WC: waist circumference; WHtR: waist to height ratio; CCL2: chemokine (C-C motif) ligand 2; HOMA-IR: homeostasis model assessment-insulin resistance; CRP: C reactive protein; VLDLc: very low density lipoprotein cholesterol; Apo: apolipoprotein. Significant differences: $P<0.05,{ }^{*}$ Pearson or ${ }^{+}$Spearman correlations test.

The individuals without IR presented a higher frequency of the phenotype $A+$ of the CCL2 polymorphism, while phenotype $A-$ is more common in the individuals with IR. These differences were also observed in genotype and allele frequencies (Table 3). Higher levels of total adiponectin as well as a parallel decrease in insulin levels and HOMA-IR index were associated with $A+$ phenotype carriers (Figure 2), as long as phenotype $A+$ was associated with low measures of BMI and BFR (Table 4). 

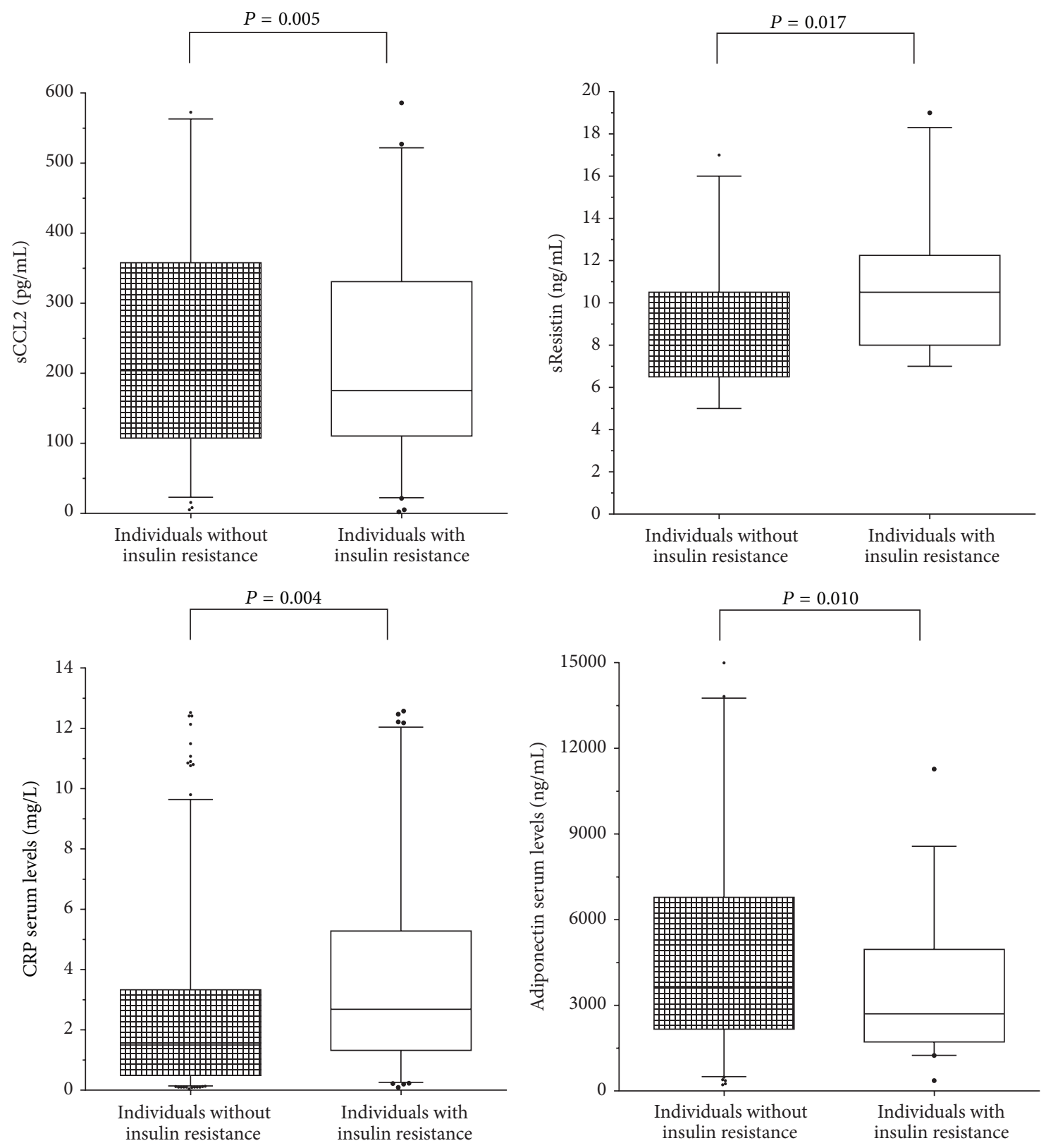

FIGURE 1: Soluble levels of adipokines and CRP by study group. $n=380$. Student's $t$-test with $P$ significantly, comparing the groups: individuals with IR versus individuals without IR.

3.4. CCR2 Polymorphism Was Not Associated with IR but Promoted Clinical Features of IR. None of the CCR2 variants had an association with IR (Table 3). The detailed analysis showed association of phenotype Ile+ with high BMI, levels of glucose and lipids (Table 4). The same as CCL2 A+ phenotype, in CCR2 Ile+ carriers presented higher levels of CCL2 compared to Ile- phenotypes (Figure 2).

3.5. A+ Phenotype of CCL2 and Ile+ of CCR2 Are Associated with High Levels of Circulating CCL2. No metabolic profile explored in this study was associated with A+ phenotype (Table 4). However, significant phenotype-by-phenotype association was observed between the CCL2-2518A allele and the CCR2 64Ile allele; carriers of the CCR2 phenotype 64Ile+ who at the same time also were phenotype A+ of CCL2 (polymorphic genotypes for the both polymorphisms) presented higher levels of soluble CCL2, than any other phenotype combinations (Figure 3 ).

\section{Discussion}

The IR is a disease of multifactorial etiology product of the interaction between the genetic component and the environment, epidemiological data describes IR as an emerging disease with epidemic proportions [19, 25, 26]. In prior information it has been postulated that there may exist 
TABLE 3: Distribution of CCL2 (G-2518A) and CCR2 (Val64Ile) gene polymorphism in Mexican-Mestizo population.

\begin{tabular}{|c|c|c|c|c|c|c|c|}
\hline \multirow{3}{*}{ Study group } & \multicolumn{3}{|c|}{ Genotype, $n(\%)$} & \multicolumn{2}{|c|}{ Phenotype, $n(\%)$} & \multicolumn{2}{|c|}{ Allele, $n(\%)$} \\
\hline & \multicolumn{7}{|c|}{ CCL2 G-2518A } \\
\hline & $\mathrm{G} / \mathrm{G}$ & G/A & $\mathrm{A} / \mathrm{A}$ & $\mathrm{A}+$ & A- & G & A \\
\hline Individuals without IR & $47(17.4)$ & $135(50.0)$ & $88(32.6)$ & $223(82.6)$ & $47(17.4)$ & $229(42.4)$ & $311(57.6)$ \\
\hline Individuals with IR & $32(29.0)$ & $51(46.4)$ & $27(24.6)$ & $78(70.9)$ & $32(29.1)$ & $115(52.3)$ & $105(47.7)$ \\
\hline${ }^{*} P$ & \multicolumn{3}{|c|}{0.01378} & \multicolumn{2}{|c|}{0.01131} & \multicolumn{2}{|c|}{0.01321} \\
\hline \multirow{3}{*}{ Study group } & \multicolumn{3}{|c|}{ Genotype, $n(\%)$} & \multicolumn{2}{|c|}{ Phenotype, $n(\%)$} & \multicolumn{2}{|c|}{ Allele, $n(\%)$} \\
\hline & \multicolumn{7}{|c|}{ CCR2 Val64Ile } \\
\hline & $\mathrm{Val} / \mathrm{Val}$ & Val/Ile & Ile/Ile & Ile+ & Ile- & Val & Ile \\
\hline Individuals without IR & $173(64.1)$ & $82(30.4)$ & $15(5.5)$ & $97(35.9)$ & $173(64.1)$ & $428(79.3)$ & $112(20.7)$ \\
\hline Individuals with IR & $67(60.9)$ & $37(33.6)$ & $6(5.5)$ & $43(39.1)$ & $67(60.9)$ & $171(77.7)$ & $49(22.3)$ \\
\hline${ }^{*} P$ & \multicolumn{3}{|c|}{0.64924} & \multicolumn{2}{|c|}{0.94889} & \multicolumn{2}{|c|}{0.63925} \\
\hline
\end{tabular}

$n=380$. IR: insulin resistance. ${ }^{*}$ Significant differences: Pearson's goodness-of-fit test $\chi^{2}$ or Fishers' exact test; $\mathrm{G}$ or Val: wild-type alleles; A+ phenotype: A/A plus G/A genotypes; Ile+ phenotype: Ile/Ile plus Ile/Val genotypes.

TABLE 4: Comparisons of body fat measurements and lipid profile between CCL2 G-2518A and CCR2 Val64Ile phenotype carriers.

\begin{tabular}{|c|c|c|c|c|c|c|}
\hline \multirow{2}{*}{ Measurements } & \multicolumn{3}{|c|}{ CCL2 G-2518A } & \multicolumn{3}{|c|}{ CCR2 Val64Ile } \\
\hline & Phenotype A+ & Phenotype A- & $P$ & Phenotype Ile+ & Phenotype Ile- & $P$ \\
\hline$n(\%)$ & $301(79)$ & $79(21)$ & & $140(37)$ & $240(63)$ & \\
\hline Weight (kg) & $72.0 \pm 15.55$ & $72.8 \pm 13.56$ & NS & $73.2 \pm 14.82$ & $71.5 \pm 15.32$ & NS \\
\hline BMI $\left(\mathrm{kg} / \mathrm{m}^{2}\right)$ & $25.9(22.9-29.1)$ & $27.5(24.3-29.65)$ & ${ }^{+} 0.028$ & $27.3(23.8-29.7)$ & $25.4(23.0-28.9)$ & $0.018^{+}$ \\
\hline Total body fat (\%) & $29.6 \pm 9.81$ & $31.7 \pm 8.57$ & NS & $31.2 \pm 9.05$ & $29.4 \pm 9.85$ & NS \\
\hline $\operatorname{BFR}\left(\mathrm{kg} / \mathrm{m}^{2}\right)$ & $7.31(5.24-9.92)$ & $8.25(6.24-11.40)$ & ${ }^{+} 0.039$ & $7.63(5.57-10.90)$ & $7.37(5.31-9.93)$ & NS \\
\hline Waist circumference $(\mathrm{cm})$ & $88.0(78.4-98.0)$ & $89.4(79.3-96.5)$ & NS & $89.1(79.5-99.0)$ & $88.1(78.3-97.6)$ & NS \\
\hline Glucose $(\mathrm{mg} / \mathrm{dL})$ & $92 \pm 12$ & $93 \pm 14$ & NS & $95 \pm 13$ & $91 \pm 12$ & $0.004^{*}$ \\
\hline Triglycerides (mg/dL) & $142 \pm 85.6$ & $146 \pm 94.2$ & NS & $156 \pm 8.5$ & $135 \pm 5.0$ & $0.039^{*}$ \\
\hline Total cholesterol (mg/dL) & $181 \pm 40.1$ & $185 \pm 34.3$ & NS & $190 \pm 43.7$ & $180 \pm 35.5$ & $0.031^{*}$ \\
\hline $\operatorname{HDLc}(\mathrm{mg} / \mathrm{dL})$ & $39 \pm 14.8$ & $37 \pm 15.8$ & NS & $38 \pm 13.6$ & $38 \pm 15.8$ & NS \\
\hline LDLc (mg/dL) & $111 \pm 35.6$ & $109 \pm 30.4$ & NS & $114 \pm 39.1$ & $108 \pm 31.2$ & NS \\
\hline VLDLc (mg/dL) & $28 \pm 16.9$ & $29 \pm 18.8$ & NS & $31 \pm 20.1$ & $26 \pm 15.3$ & $0.032^{*}$ \\
\hline Apo-A1 (mg/dL) & $114 \pm 25.1$ & $121 \pm 29.1$ & NS & $117 \pm 24.4$ & $114 \pm 27.1$ & NS \\
\hline Apo-B (mg/dL) & $112 \pm 30.0$ & $121 \pm 41.5$ & NS & $119 \pm 36.8$ & $109 \pm 27.8$ & $0.045^{*}$ \\
\hline
\end{tabular}

$n=380$. BMI: body mass index; BFR: body fat ratio; HDLc, LDLc, and VLDLc: high, low, and very low density lipoprotein cholesterol, respectively; Apo: apolipoprotein. A- or Ile-: wild-type phenotypes; polymorphic A+ phenotype (A/A plus G/A genotypes); polymorphic Ile+ phenotype (Ile/Ile plus Val/Ile genotypes). Data are presented as mean \pm standard deviation and median ( $25-75$ percentiles). ${ }^{*}$ Student's $t$-test and ${ }^{+}$Mann-Whitney $U$ test with $P$ significantly, comparing the polymorphic phenotype carriers versus wild-type phenotypes carriers.

susceptibility genes involved in adipogenesis and energy metabolism [27, 28]; in the current study we explored the distribution of polymorphisms G-2518A and Val64Ile of CCL2 and CCR2, respectively, in individuals with IR.

In addition, the individuals' carriers of different genotypes according to gene dosage were evaluated on the possible association with CCL2 levels, metabolic markers, and adiposity within the population with IR.

In the present work 380 individuals, were classified with or without IR by Stern criteria and obesity by BMI WHO criteria. The frequencies found in IR and obesity, clinical data, and anthropometric measurements are consistent with previous reports in the Mexican population [26, 29].

There are several reasons why a certain obese phenotype possibly will not be equally expressed (e.g., different physical activity levels among participants); however, the aim of the study was to investigate the relationship between the polymorphisms of CCL2 and CCR2 with CCL2 soluble levels, metabolic markers, and adiposity (like indicator of body fat status, absolute and/or relative) measured by BIA. Most studies report that the impedance method is reliable and valid. Baumgartner's contribution reviews the assumptions, applicability, equipment, measurement procedure, precision, and accuracy of the BIA method and determined that they were highly recommended [30-32]. Unfortunately we did not evaluate the fat distribution in study subjects; hence, we were not able to include a body fat distribution analysis.

In this set, clinic profile and the ratio of prevalence of IR, presented in the present study, suggest that IR is a complex disease, meaning that different phenotypes are observed with various clinical stages during the development of the pathogenic process. It has been postulated that in 

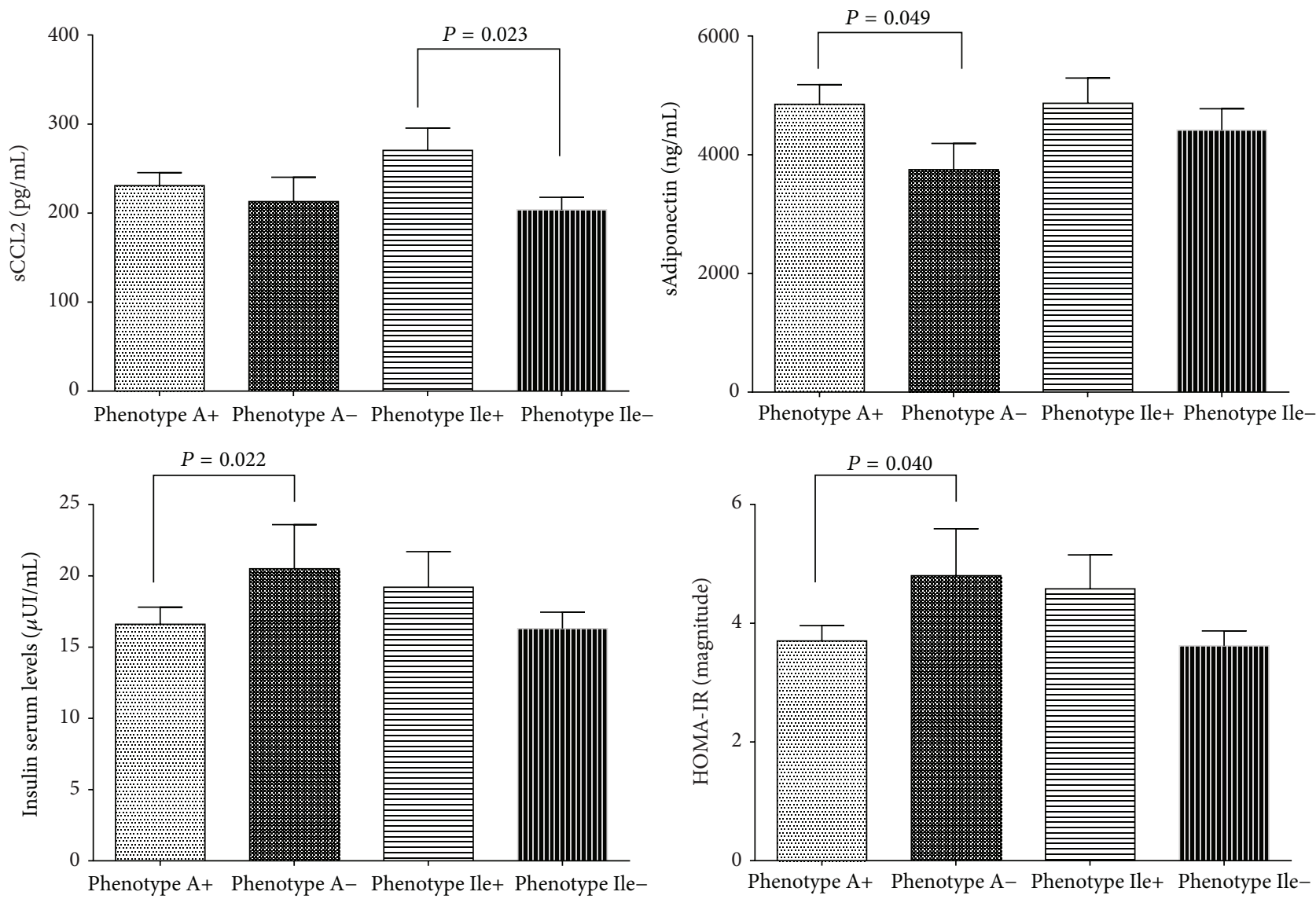

Figure 2: Soluble levels of adipokines, insulin, and HOMA-IR by CCL2 G-2518A and CCR2 Val64Ile phenotype carriers. $n=380$. A+ phenotype: A/A plus G/A genotypes; Ile+ phenotype: Ile/Ile plus Val/Ile genotypes. Student's $t$-test with $P$ significantly, comparing the polymorphic phenotype carriers versus wild-type phenotypes carriers.

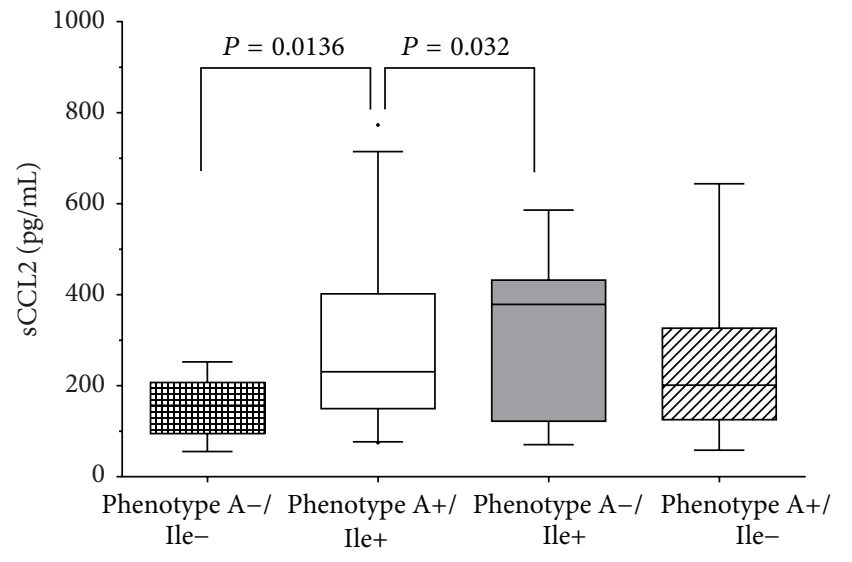

FIgUre 3: Soluble levels of CCL2 by phenotypes. Phenotype A-/Ile(genotypes GG/ValVal) $n=48$; phenotype A+/Ile+ (genotypes AA plus GA/ValIle plus IleIle) $n=108$; phenotype A-/Ile+ (genotypes GG/ValIle plus IleIle) $n=32$; phenotype A+/Ile- (genotypes AA plus GA/ValVal) $n=192$. One way ANOVA and Tukey's post hoc test with $P$ significantly, comparing the double-polymorphic phenotype carriers, $(\mathrm{A}+/ \mathrm{Ile}+)$ versus double-wild-type phenotype carriers (A-/Ile-). course of the natural history of the IR, the hallmark is a lowgrade subclinical inflammatory process, in which circulating monocytes infiltrate to adipose tissue, in a redundant manner, and polarize to M1 macrophages, becoming the main producers of chemokines and their receptors [33].

Two important observations lead this study to supporting that adiposity is associated with metabolic markers, in IR development. First, increased adiposity indicators and triglyceride levels were observed, and secondly no differences were found in other components of lipid profile. The first results are in agreement with previous studies [21, 33-35] that was attributed to the presence of obesity; in this case the expansion and accumulation of fat promotes the progress to IR. The second point given by the present study can be explained by the fact that IR is just component during the development of a mayor disease, since dyslipidemia is a later event, that could generate metabolic syndrome [36].

We observe that IR individuals presented higher inflammatory state, due to increased CCL2 levels in contrast to individuals without IR. These results can be explained by the increased levels of expression of adipokines, chemokines, and proinflammatory cytokines associated with a parallel increase 
in the number, macrophages in the adipose tissue. The later ones, mainly macrophages with M1 phenotype, are crucial in IR, based on the fact that these cells are the important source of proinflammatory markers: TNF- $\alpha$, IL-6, and CRP $[2,33,37]$.

Alongside, in the individuals with IR was confirmed the presence of a low-grade inflammatory process represented by the increased levels of sResistin, CRP, and the decreased levels of total adiponectin and the correlation of them with adiposity status, parallel to the increase in BMI, BFR, and metabolic markers.

The importance of the present results lies in the biological relationship that exists between IR and obesity for the development of other diseases, for instance, metabolic syndrome. Insulin has the following functions: on one side, it promotes synthesis of insulin-like growth factor 1 , which correlates with fat accumulation, and increase of white adipose tissue; on other side, it is the leader in the deregulations in the secretion of adipokines and metabolic markers that has been associated with certain types of cancer and other chronic diseases such as T2DM $[1,4,6,38]$.

The main pathological mechanism that connect the increase of adipose tissue with IR is the disfunction of the immune system and the establishment of a low-grade subclinical systemic chronic inflammation state, as a result of increased adiposity. This dysregulation of the immune system can be explained on two mechanisms: first, the adipose tissue in obese subjects has an increased amount of infiltrated macrophages who present an M1 phenotype and have an increase in the expression of 4 retinol binding protein (RPB4); and second, it has been found that high concentrations of fatty acids and RPB4 induce the expression of TNF- $\alpha$ and the signaling mediated by the toll-like receptor $4[1,27]$.

In this context, the inflammatory process in IR is an underlying clinical sign in the course of the disease and is identified by an increase in levels of inflammatory markers, as well as deregulation in the production of adipokines; in this respect there is evidence based on animal model studies and in humans, in which the important role of white adipose tissue is demonstrated in the maintenance of an inflammatory response associated with the development of IR [6].

There are numerous studies that have found interactions between polymorphisms and development of diseases such as metabolic syndrome. Due to the link of inflammation with development of IR, numerous genes have been studied [2, 28]. The chemokine CCL2 and their CCR2 receptor have been studied in recent years because of their involvement in the recruitment of macrophages to adipose tissue and subsequent differentiation into proinflammatory M1 phenotype [27, 33, 39].

The polymorphism G-2518A, in CCL2 gene, was identified as a $\mathrm{G}$ to $\mathrm{A}$ transition; this change is near to a response site of NF-kB and has been speculated that increases affinity to its ligand; this results in increased levels of CCL2 in the bloodstream and further recruitment of macrophages to adipose tissue compared to those individuals exhibiting wildtype allele. Concerning polymorphism in CCR2, Val64Ile, is a transition $\mathrm{G}$ to $\mathrm{A}$ at position 190 of CCR2, changing valine codon (GTC) to isoleucine (ATC) at position 64, a conservative change of neutral nonpolar amino acids. This change makes the CCR2A isoform more stable and increases its half-life but does not affect the stability of the isoform CCR2B $[1,40]$.

On the stage of genetic diversity between populations, the reported frequencies for polymorphic alleles $-2518 \mathrm{~A}$ and 64Ile ranging from $39.1 \%$ to $83.2 \%$ and $9.5 \%$ to $25.6 \%$, respectively, with differences with the frequencies reported in this study (Tables 5(a) and 5(b)), show that wild-type allele changes in some populations, such as Asiatic. In the present study the allele polymorphic frequencies are similar to those reported in Mexican-Mestizos by González-Enríquez et al. and different from Vázquez-Lavista et al. [41, 42].

We found differences when comparing frequencies of alleles of our Mexican population with other populations of different nations, for example, the Asiatic, Caucasian European, or American populations, with a different genetic background, which is explained by the distribution of alleles as result of their anthropological relationships. Since the conquest by the Spanish, European genetics was introduced on the natures, over the centuries. Hence, individuals included in the present study were not pure endogenous Mexican population. Therefore the differences in genotype distribution with other Mexican populations may be because of the fact that in our study group participants were characterized as MexicanMestizos of Western Mexico (Tables 5(a) and 5(b)).

In this study group, CCL2 polymorphism was associated with IR, according to the results showing differences in the distribution of frequencies of genotypes, phenotypes, and alleles, with a higher contribution of the A+ phenotype than A- phenotype for the presence of IR. A+ phenotype was associated with higher levels of total sAdiponectin and lower levels of insulin and HOMA-IR magnitude, while a decrease was shown in BMI and BFR. The opposite was observed for individuals carrying of polymorphic phenotype Ile+; they had an increase in lipid profile; these results show influence of both polymorphisms in the accumulation of body fat and its relation to the metabolic status of individuals.

This can be explained because this is the main tissue involved in the production of adipokines and increases its volume in the presence of a proinflammatory environment $[5,33]$. Previous studies have demonstrated the interference of proinflammatory cytokine in the signaling pathway of insulin receptor [27]. Parallel to this stage, we observed increased levels of glucose in individuals with phenotype Ile+ which indicates involvement of this polymorphism in the establishment of IR.

The adiposity status shown in the individuals of this study regarding the polymorphisms in CCL2 and CCR2 and its association with metabolic and inflammatory markers, and levels of adipokines have not been previously described for Mexican-Mestizo population.

Among the most important results of this study are that $\mathrm{A}+$ phenotype of CCL2 and Ile+ of CCR2 carriers are associated with higher levels of circulating CCL2; this difference is attributed to the fact that this variant generates a more stable form of the receptor CCR2 membrane which has a longer half-life, allowing for longer signaling that in people with wild-type polymorphism variant [40]. 
TABLE 5: (a) Distribution of G-2518A polymorphism in CCL2 gene in other populations. (b) Distribution of Val64Ile polymorphism in CCR2 gene in other populations.

(a)

\begin{tabular}{|c|c|c|c|c|c|c|c|c|}
\hline Author & Population & $n$ & G & A & $\mathrm{G} / \mathrm{G}$ & $\mathrm{A} / \mathrm{G}$ & $\mathrm{A} / \mathrm{A}$ & $P$ \\
\hline The present study & Mexican-Mestizo & 380 & 45.2 & 54.6 & 20.7 & 48.9 & 30.3 & - \\
\hline Vázquez-Lavista et al. [41] & Mexican-Mestizo & 126 & 57.5 & 42.5 & 29.4 & 56.3 & 14.3 & $<0.001$ \\
\hline González-Enríquez et al. [42] & Mexican-Mestizo & 21 & 52.4 & 47.6 & 27.0 & 50.0 & 23.0 & 0.370 \\
\hline Bektas-Kayhan et al. [24] & Caucasian & 140 & 16.8 & 83.2 & 0.8 & 32.1 & 67.1 & $<0.001$ \\
\hline Kruszyna et al. [43] & Caucasian & 323 & 29.9 & 70.1 & 7.4 & 44.9 & 47.7 & $<0.001$ \\
\hline Kucukgergin et al. [44] & Caucasian & 197 & 30.2 & 69.8 & 9.1 & 42.1 & 48.7 & $<0.001$ \\
\hline Kouyama et al. [13] & Asian & 361 & 34.6 & 65.4 & - & - & - & - \\
\hline Singh et al. [7] & Asian & 200 & 35.3 & 64.7 & 11.0 & 48.5 & 40.5 & $<0.001$ \\
\hline Mandal et al. [45] & Asian & 390 & 37.2 & 62.8 & 13.2 & 48.0 & 38.8 & 0.001 \\
\hline Chen et al. [23] & Asian & 344 & 51.7 & 48.3 & 26.7 & 50.0 & 23.3 & 0.015 \\
\hline Wu et al. [46] & Asian & 253 & 60.1 & 39.1 & 34.8 & 52.2 & 13.0 & $<0.001$ \\
\hline
\end{tabular}

Pearson's goodness-of-fit test $\chi^{2}$ or Fishers' exact test with $P$ significantly, comparing alleles and/or genotypes polymorphism distribution in Mexican-Mestizos population in this study versus distribution in other populations. G wild-type allele, A polymorphic allele.

(b)

\begin{tabular}{|c|c|c|c|c|c|c|c|c|}
\hline Author & Population & $n$ & Val & Ile & Val/Val & Val/Ile & Ile/Ile & $P$ \\
\hline The present study & Mexican-Mestizo & 380 & 78.8 & 21.2 & 63.2 & 31.3 & 5.5 & - \\
\hline Vázquez-Lavista et al. [41] & Mexican-Mestizo & 126 & 75.8 & 24.2 & 58.7 & 34.1 & 7.2 & 0.330 \\
\hline González-Enríquez et al. [42] & Mexican-Mestizo & 21 & 90.5 & 9.5 & 82.6 & 17.3 & 0 & 0.006 \\
\hline Bektas-Kayhan et al. [24] & Caucasians & 140 & 88.5 & 11.42 & 80.0 & 17.1 & 2.9 & $<0.001$ \\
\hline Kucukgergin et al. [44] & Caucasians & 197 & 89.6 & 10.4 & 80.7 & 17.8 & 1.5 & $<0.001$ \\
\hline González et al. [47] & Caucasians & 280 & 90.0 & 10.0 & 80.0 & 19.0 & 1.0 & $<0.001$ \\
\hline Mandal et al. [45] & Asian & 390 & 74.4 & 25.6 & 54.8 & 39.2 & 6.0 & 0.046 \\
\hline Wu et al. [46] & Asian & 253 & 80.6 & 19.4 & 64.4 & 32.4 & 3.2 & 0.438 \\
\hline Singh et al. [7] & Asian & 200 & 80.5 & 19.5 & 63.0 & 35.0 & 2.0 & 0.501 \\
\hline Zandifar et al. [48] & Asian & 100 & 87.5 & 12.5 & 75.0 & 25.0 & 0 & 0.007 \\
\hline Chen et al. [23] & Asian & 344 & 89.1 & 10.9 & 80.2 & 17.7 & 2.1 & $<0.001$ \\
\hline
\end{tabular}

Pearson's goodness-of-fit test $\chi^{2}$ or Fishers' exact test with $P$ significantly, comparing alleles and/or genotypes polymorphism distribution in Mexican-Mestizos population in this study versus distribution in other populations. Val: wild-type allele; Ile: polymorphic allele.

Taking all results together, the present work shows new evidence that suggests that genetic factors contribute to the development of IR and this triggers pathobiological processes. These changes could influence the clinical course and severity of obesity-related diseases, such as IR. Therefore, the genetic load on individuals could play an important role in the genesis of diseases observed in obese subjects.

\section{Conclusions}

The most important results in the individuals of the present study are summarized as follows: association of adiposity with metabolic markers alongside with inflammatory state, CCL2 polymorphism is associated with IR, while CCR2 was associated with clinical features of IR besides it was seen that the individuals that had both polymorphic phenotypes had increased levels of CCL2.

These data suggests that the CCL2A+ phenotype could impact the reduced body fat storage, metabolic and healthy adipokine profile in Mexican-Mestizo individuals. The opposite, CCR264Ile+ phenotype is associated with altered profile of metabolic markers and BMI. All this data suggests that the CCL2 and CCR2 polymorphisms and the signaling through this interactions could play a role in the metabolic changes associated with IR in Mexican-Mestizo population.

As a final remark we can conclude that an increase of CCL2 serum levels is associated with the polymorphic phenotypes $(\mathrm{A}+/ \mathrm{Ile}+)$ of the polymorphisms $\mathrm{G}-2518 \mathrm{~A}$ in CCL2 and Val64Ile in CCR2 in individuals with insulin resistance of Mexican population.

\section{Conflict of Interests}

The authors declare that there is no conflict of interests regarding the publication of this paper.

\section{Authors' Contribution}

Milton-Omar Guzmán-Ornelas and Marcelo Heron Petri equally contributed to this work. 


\section{Acknowledgment}

This work was supported by Grant no. PS-2009-552 to RosaElena Navarro-Hernández of the State Council of Science and Technology (COECyTJal-University of Guadalajara).

\section{References}

[1] T. Ota, "Chemokine systems link obesity to insulin resistance," Diabetes \& Metabolism Journal, vol. 37, no. 3, pp. 165-172, 2013.

[2] C. de Luca and J. M. Olefsky, "Inflammation and insulin resistance," FEBS Letters, vol. 582, no. 1, pp. 97-105, 2008.

[3] R. A. Bastarrachea, J. C. López-Alvarenga, V. E. BoladoGarcía, J. Téllez-Mendoza, H. Laviada-Molina, and A. G. Comuzzie, "Macrophages, inflammation, adipose tissue, obesity and insulin resistance," Gaceta Médica de México, vol. 143, no. 6, pp. 505-512, 2007.

[4] T. O'Connor, L. Borsig, and M. Heikenwalder, "CCL2-CCR2 signaling in disease pathogenesis," Endocrine, Metabolic \& Immune Disorders-Drug Targets, vol. 15, no. 2, pp. 105-118, 2015.

[5] B. Gustafson, "Adipose tissue, inflammation and atherosclerosis," Journal of Atherosclerosis and Thrombosis, vol. 17, no. 4, pp. 332-341, 2010.

[6] A. Ueda, Y. Ishigatsubo, T. Okubo, and T. Yoshimura, "Transcriptional regulation of the human monocyte chemoattractant protein-1 gene: cooperation of two NF- $\kappa \mathrm{B}$ sites and NF- $\kappa \mathrm{B} / \mathrm{Rel}$ subunit specificity," Journal of Biological Chemistry, vol. 272, no. 49, pp. 31092-31099, 1997.

[7] V. Singh, P. Srivastava, N. Srivastava, R. Kapoor, and R. D. Mittal, "Association of inflammatory chemokine gene CCL2I/D with bladder cancer risk in North Indian population," Molecular Biology Reports, vol. 39, no. 10, pp. 9827-9834, 2012.

[8] T. Yoshimura and J. J. Oppenheim, "Chemokine-like receptor 1 (CMKLR1) and chemokine (C-C motif) receptor-like 2 (CCRL2); two multifunctional receptors with unusual properties," Experimental Cell Research, vol. 317, no. 5, pp. 674-684, 2011.

[9] A. M. Valdes, M. L. Wolfe, E. J. O’Brien et al., "Val64Ile polymorphism in the $\mathrm{C}-\mathrm{C}$ chemokine receptor 2 is associated with reduced coronary artery calcification," Arteriosclerosis, Thrombosis, and Vascular Biology, vol. 22, no. 11, pp. 1924-1928, 2002.

[10] J. Petrkova, Z. Cermakova, J. Drabek, J. Lukl, and M. Petrek, "CC chemokine receptor (CCR)2 polymorphism in Czech patients with myocardial infarction," Immunology Letters, vol. 88, no. 1, pp. 53-55, 2003.

[11] K. Chatterjee, C. Dandara, M. Hoffman, and A.-L. Williamson, "CCR2-V64I polymorphism is associated with increased risk of cervical cancer but not with HPV infection or pre-cancerous lesions in African women," BMC Cancer, vol. 10, article 278, 2010.

[12] E. Simeoni, M. M. Hoffmann, B. R. Winkelmann et al., "Association between the A-2518G polymorphism in the monocyte chemoattractant protein-1 gene and insulin resistance and Type 2 diabetes mellitus," Diabetologia, vol. 47, no. 9, pp. 1574-1580, 2004.

[13] K. Kouyama, K. Miyake, M. Zenibayashi et al., "Association of serum MCP-1 concentration and MCP-1 polymorphism with insulin resistance in Japanese individuals with obese type 2 diabetes," Kobe Journal of Medical Sciences, vol. 53, no. 6, pp. 345-354, 2007.

[14] W. R. Bolus, D. A. Gutierrez, A. J. Kennedy, E. K. AndersonBaucum, and A. H. Hasty, "CCR2 deficiency leads to increased eosinophils, alternative macrophage activation, and type 2 cytokine expression in adipose tissue," Journal of Leukocyte Biology, vol. 98, no. 4, pp. 467-477, 2015.

[15] S. E. Stern, K. Williams, E. Ferrannini, R. A. DeFronzo, C. Bogardus, and M. P. Stern, "Identification of individuals with insulin resistance using routine clinical measurements," Diabetes, vol. 54, no. 2, pp. 333-339, 2005.

[16] DOF 07-02-1984, ú.r.D., Reglamento de la Ley General de Salud en Materia de Investigación para la Salud.

[17] R. Ness-Abramof and C. M. Apovian, "Waist circumference measurement in clinical practice," Nutrition in Clinical Practice, vol. 23, no. 4, pp. 397-404, 2008.

[18] WHO, "Physical status: the use and interpretation of anthropometry. Report of a WHO Expert Committee," World Health Organization Technical Report Series 854, WHO, 1995.

[19] World Health Organization, Obesity: Preventing and Managing the Global Epidemic, World Health Organization, Geneva, Switzerland, 1st edition, 2012.

[20] R. Fernandez-Vazquez, Á. Millán Romero, M. Á. Barbancho, and J. R. Alvero-Cruz, "Abdominal bioelectrical impedance analysis and anthropometry for predicting metabolic syndrome in middle aged men," Nutrición Hospitalaria, vol. 32, no. 3, pp. 1122-1130, 2015.

[21] P. Deurenberg, M. Yap, and W. A. van Staveren, "Body mass index and percent body fat: a meta analysis among different ethnic groups," International Journal of Obesity, vol. 22, no. 12, pp. 1164-1171, 1998.

[22] S. A. Miller, D. D. Dykes, and H. F. Polesky, "A simple salting out procedure for extracting DNA from human nucleated cells," Nucleic Acids Research, vol. 16, no. 3, p. 1215, 1988.

[23] M.-K. K. Chen, K.-T. Yeh, H.-L. Chiou, C.-W. Lin, T.-T. Chung, and S.-F. Yang, "CCR2-64I gene polymorphism increase susceptibility to oral cancer," Oral Oncology, vol. 47, no. 7, pp. 577$582,2011$.

[24] K. Bektas-Kayhan, M. Unur, Z. Boy-Metin, and B. Cakmakoglu, "MCP-1 and CCR2 gene variants in oral squamous cell carcinoma," Oral Diseases, vol. 18, no. 1, pp. 55-59, 2012.

[25] OMS, Obesity: Preventing and Managing the Global Epidemic, OMS, 1st edition, 2012.

[26] G. Olaiz-Fernández, J. Rivera, T. Shamah et al., Encuesta Nacional de Salud y Nutrición 2006, Instituto Nacional de Salud Pública, 2006.

[27] A. Rull, J. Camps, C. Alonso-Villaverde, and J. Joven, "Insulin resistance, inflammation, and obesity: role of monocyte chemoattractant protein-1 (orCCL2) in the regulation of metabolism," Mediators of Inflammation, vol. 2010, Article ID 326580, 11 pages, 2010.

[28] M. L. R. Curti, P. Jacob, M. C. Borges, M. M. Rogero, and S. R. G. Ferreira, "Studies of gene variants related to inflammation, oxidative stress, dyslipidemia, and obesity: implications for a nutrigenetic approach," Journal of Obesity, vol. 2011, Article ID 497401, 31 pages, 2011.

[29] J. P. Gutiérrez, J. Rivera, T. Shamah, C. Oropez, and M. H. Ávila, Encuesta Nacional de Salud y Nutrición 2012. Resultados Nacionales, Instituto Nacional de Salud Pública, Cuernavaca, México, 2012.

[30] D. Brodie, V. Moscrip, and R. Hutcheon, "Body composition measurement: a review of hydrodensitometry, anthropometry, and impedance methods," Nutrition, vol. 14, no. 3, pp. 296-310, 1998. 
[31] U. G. Kyle, I. Bosaeus, A. D. de Lorenzo et al., "Bioelectrical impedance analysis-part II: utilization in clinical practice," Clinical Nutrition, vol. 23, no. 6, pp. 1430-1453, 2004.

[32] M. Y. Jaffrin and H. Morel, "Body fluid volumes measurements by impedance: a review of bioimpedance spectroscopy (BIS) and bioimpedance analysis (BIA) methods," Medical Engineering and Physics, vol. 30, no. 10, pp. 1257-1269, 2008.

[33] B. Feng, T. Zhang, and H. Xu, "Human adipose dynamics and metabolic health," Annals of the New York Academy of Sciences, vol. 1281, no. 1, pp. 160-177, 2013.

[34] C. T. Lichtash, J. Cui, X. Guo et al., "Body adiposity index versus body mass index and other anthropometric traits as correlates of cardiometabolic risk factors," PLoS ONE, vol. 8, no. 6, Article ID e65954, 2013.

[35] F. Taverne, C. Richard, P. Couture, and B. Lamarche, "Abdominal obesity, insulin resistance, metabolic syndrome and cholesterol homeostasis," PharmaNutrition, vol. 1, no. 4, pp. 130-136, 2013.

[36] A. V. Castro, C. M. Kolka, S. P. Kim, and R. N. Bergman, "Obesity, insulin resistance and comorbidities? Mechanisms of association," Arquivos Brasileiros de Endocrinologia e Metabologia, vol. 58, no. 6, pp. 600-609, 2014.

[37] M. C. Morrison and R. Kleemann, "Role of macrophage migration inhibitory factor in obesity, insulin resistance, type 2 diabetes, and associated hepatic co-morbidities: a comprehensive review of human and rodent studies," Frontiers in Immunology, vol. 6, article 308, 2015.

[38] L. Yao, O. Herlea-Pana, J. Heuser-Baker, Y. Chen, and J. BarlicDicen, "Roles of the chemokine system in development of obesity, insulin resistance, and cardiovascular disease," Journal of Immunology Research, vol. 2014, Article ID 181450, 11 pages, 2014.

[39] S. L. Deshmane, S. Kremlev, S. Amini, and B. E. Sawaya, "Monocyte chemoattractant protein-1 (MCP-1): an overview," Journal of Interferon and Cytokine Research, vol. 29, no. 6, pp. 313-325, 2009.

[40] Y. Huang, H. Chen, J. Wang et al., "Relationship between CCR2V64I polymorphism and cancer risk: a meta-analysis," Gene, vol. 524, no. 1, pp. 54-58, 2013.

[41] L. G. Vázquez-Lavista, G. Lima, F. Gabilondo, and L. Llorente, "Genetic association of monocyte chemoattractant protein 1 (MCP-1)-2518 polymorphism in Mexican patients with transitional cell carcinoma of the bladder," Urology, vol. 74, no. 2, pp. 414-418, 2009.

[42] G. V. González-Enríquez, M. I. Rubio-Benítez, V. GarcíaGallegos, E. Portilla-de Buen, R. Troyo-Sanromán, and C. Á. Leal-Cortés, "Contribution of TNF-308A and CCL2-2518A to carotid intima-media thickness in obese mexican children and adolescents," Archives of Medical Research, vol. 39, no. 8, pp. 753759, 2008.

[43] Ł. Kruszyna, M. Lianeri, B. Rubis et al., "CCL2-2518 A/G single nucleotide polymorphism as a risk factor for breast cancer," Molecular Biology Reports, vol. 38, no. 2, pp. 1263-1267, 2011.

[44] C. Kucukgergin, F. K. Isman, S. Dasdemir et al., "The role of chemokine and chemokine receptor gene variants on the susceptibility and clinicopathological characteristics of bladder cancer," Gene, vol. 511, no. 1, pp. 7-11, 2012.

[45] R. K. Mandal, T. Agrawal, and R. D. Mittal, "Genetic variants of chemokine CCL2 and chemokine receptor CCR2 genes and risk of prostate cancer," Tumor Biology, vol. 36, no. 1, pp. 375381, 2015.
[46] H.-H. H. Wu, T.-H. Lee, Y.-T. Tee et al., "Relationships of single nucleotide polymorphisms of monocyte chemoattractant protein 1 and chemokine receptor 2 with susceptibility and clinicopathologic characteristics of neoplasia of uterine cervix in Taiwan women," Reproductive Sciences, vol. 20, no. 10, pp. 1175-1183, 2013.

[47] P. González, R. Alvarez, A. Batalla et al., "Genetic variation at the chemokine receptors CCR5/CCR2 in myocardial infarction," Genes and Immunity, vol. 2, no. 4, pp. 191-195, 2001.

[48] A. Zandifar, M. Taheriun, S. Soleimani, F. Haghdoost, M. Tajaddini, and S. H. Javanmard, "Investigation of chemokine receptor CCR2V64Il gene polymorphism and migraine without aura in the iranian population," The Scientific World Journal, vol. 2013, Article ID 836309, 5 pages, 2013. 


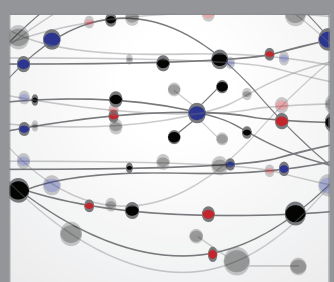

The Scientific World Journal
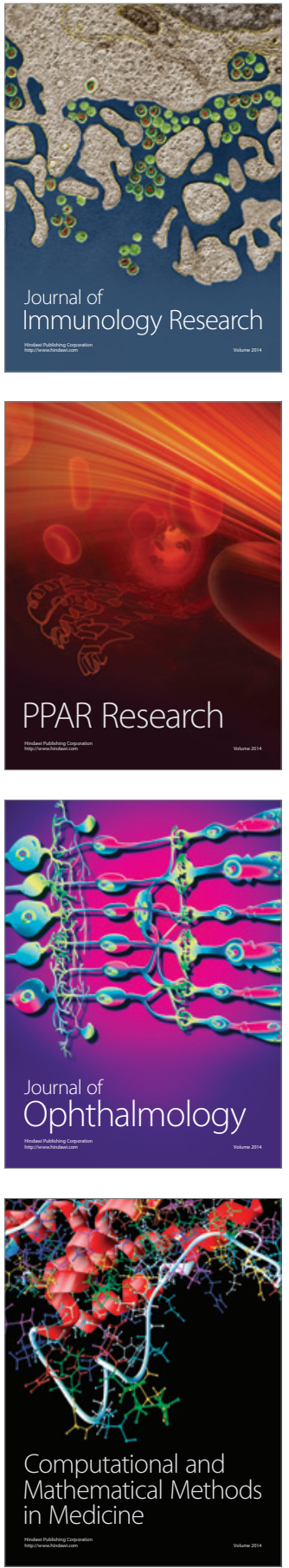

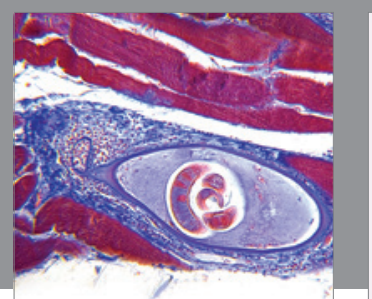

Gastroenterology Research and Practice

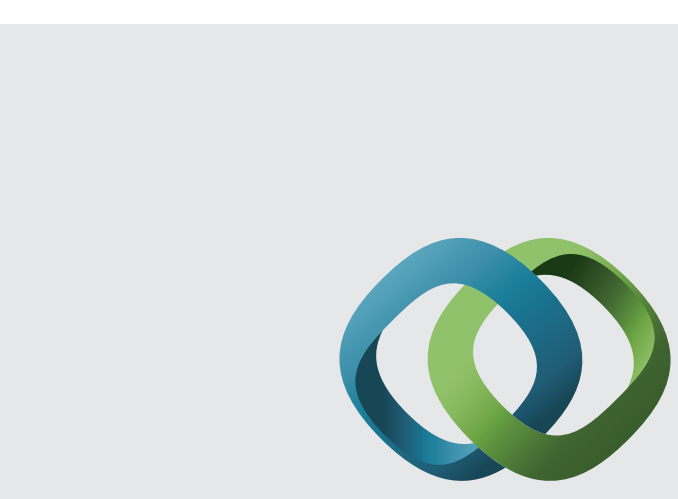

\section{Hindawi}

Submit your manuscripts at

http://www.hindawi.com
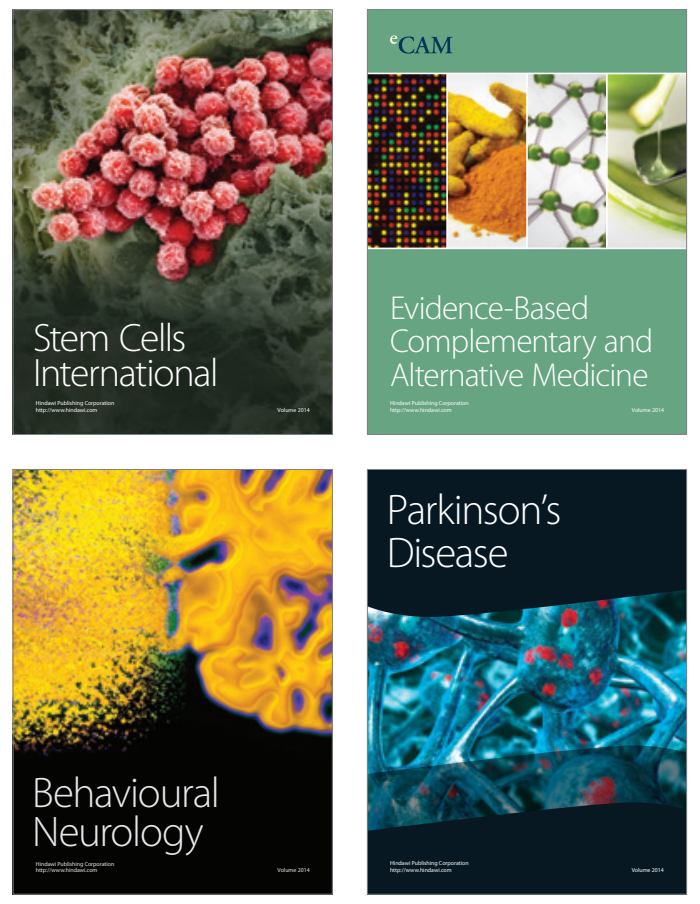
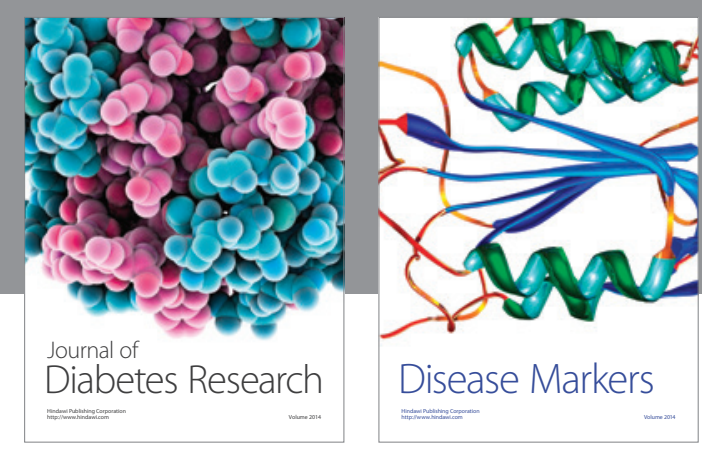

Disease Markers
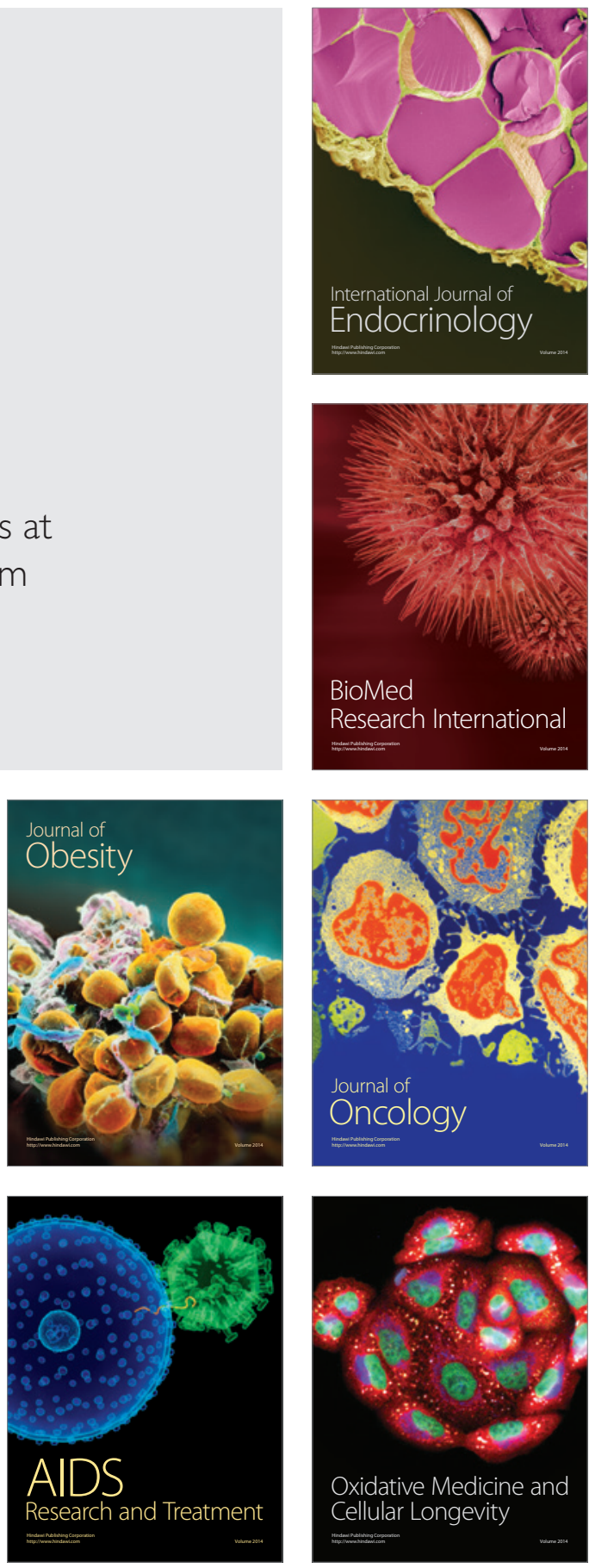